\title{
ON THE MATRICES THAT PRESERVE THE VALUE OF THE IMMANANT OF THE UPPER TRIANGULAR MATRICES
}

\author{
RosÁRio FERnANDES AND HENRIQUe F. DA CRUZ
}

Abstract. Let $\chi$ be an irreducible character of the symmetric group of degree $n$, let $M_{n}(\mathbb{F})$ be the linear space of $n$-square matrices with elements in the field $\mathbb{F}$ of characteristic zero, let $T_{n}^{U}(\mathbb{F})$ be the subset of $M_{n}(\mathbb{F})$ of the upper triangular matrices and let $d_{\chi}$ be the immanant associated with $\chi$. We denote by $\mathscr{T}\left(S_{n}, \chi\right)$ the set of all $A \in M_{n}(\mathbb{F})$, such

$$
d_{\chi}(A X)=d_{\chi}(X)
$$

for all $X \in T_{n}^{U}(\mathbb{F})$. The purpose of this paper is to present, in some cases, a complete description of the matrices in the set $\mathscr{T}\left(S_{n}, \chi\right)$ (2000): 15A15.

Keywords and phrases: Matrix preservers, immanants, triangular matrix.

\section{REFERENCES}

[1] H. Boerner, Representation of Groups, American Elsevier, New York, 1970.

[2] M. A. DUFFNER, A note on singuler matrices that satisfying certain polynomial identities, Linear and Mult. Algebra, 42 (1997), 213-219.

[3] R. FERnANDES, Matrices that preserve the value of the generalized matrix function of the upper triangular matrices, Linear Algebra Appl., 401 (2005), 47-65.

[4] G. JAMES AND A. KeRBER, The representation theory of the symmetric group, Addison-Wesley, 1981.

[5] M. Marcus And J. Chollet, Decomposable symmetrized tensors, Linear and Mult. Algebra, 6 (1978), 317-326.

[6] M. Marcus AND J. ChOllet, Linear groups defined by decomposable tensor equality, Linear and Mult. Algebra, 8 (1980), 207-212.

[7] M. MARCUS, Decomposable symmetric tensors and an extended LR decomposition theorem, Linear and Mult. Algebra, 6 (1978), 327-330.

[8] R. MerRis, Equality of decomposable symmetrized tensors, Can.J. Math. XXVIII, 5 (1975), 1022-1024.

[9] R. Merris, Multilinear Algebra, Gordon and Breach, Amesterdam, 1997.

[10] J. A. Dias DA SiLva, Conditions for equality of decomposable symmetric tensors, Linear Algebra Appl., 24 (1979), 85-92.

[11] G. N. De Oliveira, Generalized matrix functions, Estudos do Instituto Gulbenkian de Ciência, Oeiras, Portugal, 1973.

[12] G. N. De Oliveira And J. A. Dias Da Silva, Conditions for equality of decomposable symmetric tensors II, Linear Algebra Appl., 28 (1979), 161-176.

[13] G. N. De Oliveira, A. P. Santana And J. A. Dias Da Silva, A note on the equality of star products, Linear and Mult. Algebra, 14 (1983), 157-163.

[14] G. N. DE Oliveira, Interlacing inequalities. Matrix groups, Linear Algebra Appl., 162-164 (1992), 297-307.

[15] G. N. DE Oliveira AND J. A. Dias Da Silva, Equality of decomposable symmetrized tensors and *-matrix groups, Linear Algebra Appl., 49 (1983), 191-219. 